\title{
How Botulinum Toxin Type A- Occupational Therapy (OT)-Functional Electrical Stimulation (FES) Modify Spasticity and Functional Recovery in Patients with Upper Limb Spasticity Post Stroke
}

Marvulli R. ${ }^{1,}$, , Mastromauro L. ${ }^{1}$, Romanelli E. ${ }^{1}$, Lopopolo A. ${ }^{1}$, Dargenio M. ${ }^{1}$, Fornarelli F. ${ }^{1}$, Conte E. ${ }^{2}$, Fiore P. ${ }^{1}$, Megna M. ${ }^{1}$ and Ianieri G. ${ }^{1}$

\author{
${ }^{1}$ Department of Basic Sciences, Neurosciences and Sense Organs- Physical Medicine and Rehabilitation and Spinal \\ Unit Unipolar-University of Bari “Aldo Moro"-Piazza Giulio Cesare n 11, 70125 Bari, Italy and ${ }^{2}$ School of Advanced \\ International Studies on Applied Theoretcial and non Linear Methodologies of Physics-Bari
}

\begin{abstract}
Introduction: Spasticity is the most important problem in the recovery of a satisfactory function of the upper limb to the patient outcomes of cerebral stroke. Objective of the study is to compare the results in functional recovery in patients treated with botulinum toxin A and occupational therapy and patients treated with botulinumtoxinA, occupational therapy (OT) and functional electrical stimulation (FES) (Ness H-200).

Materials and methods: 36 patients (middle aged 55,25 $\pm 6,5$ ) with spasticity of the upper limbs for more than six months and in particular with the involvement of the muscles of the hand. Patients were divided into two groups: Group I underwent

Marvulli R. botulinumtoxinA-occupational therapy-functional electrical stimulation, Group II underwent botulinumtoxinA and occupational therapy. All patients were evaluated with the modified Ashworth scale, with the measurement of the passive ROM, the evaluation of the amplitude of the compound action potential of the median nerve (registration site in the flexor digitorum superficial) and ARAT test at the time T0 (recruitment and infiltration), T1 (20 days after first infiltration), T2 (3 months after first infiltration), T3 (four months after first infiltration; we reinjected botulinumtoxinA), T4 (20 days after second infiltration), T5 (3 months after second infiltration), T6 ( four months after first infiltration; we reinjected botulinum toxin $\mathrm{A})$.
\end{abstract}

Result: Group treated with botulinumtoxinA-OT-FES showed a statistically significant improvement compared to the other group in the evaluation of the passive ROM, in the assessment of spasticity with the modified Ashworth scale, in the reduction of the amplitude cMAP of the median nerve and in the score of ARAT test.

Conclusions: The application of the FES joined to treatment with botulinumtoxinA and OT proves effective synergy for greater functional recovery of the upper limb and the best possible outcomes.

Keywords: Stroke, spasticity, functional electrical stimulation (FES), myometry, botulinum toxin type A, physiotherapy.

\section{INTRODUCTION}

Stroke is one of the leading causes of disability in modern world. Damage to the pyramidal tract gives rise to a set of positive and negative signs called the upper moto neuron syndrome [1-2]. Positive signs include hyper-excitability of the stretch reflex, synkinesias, muscle co-contraction, increased muscle stiffness, involuntary movements and dysto

*Address correspondence to this author at the Department of Basic Sciences, Neurosciences and Sense Organs- Physical Medicine and Rehabilitation and Spinal Unit Unipolar-University of Bari "Aldo Moro"-Piazza Giulio Cesare n 11, 70125 Bari, Italy; E-mail: ricmarv81@hotmail.it; elio.conte@fastwebnet.it pia. Therefore spasticity is the most common sign of stroke in those surviving to first months after first-ever stroke [3]. It results in increased, involuntary, velocity-dependent muscle tone that causes resistance to passive movement [2]. Spasticity can cause problems performing normal daily activities, especially evident for patients affecting the upper limb, with its social cost and loss productivity [4-5]. In fact, $15-30 \%$ of stroke survivors not have fully recovered hand function, limiting patient's autonomy to perform most of activities of daily living [6]. In this abnormal motor state, there is increased tone in antigravity muscles, which muscles flexing the fingers, reducing muscle control to use the hand and arm. The development of compensatory strategies to perform the 
movements and the non use of affected limb aggravates clinical conditions [7]. It is well recognized that spasticity may interfere with motor and activity performance, cause pain and lead to secondary complications [8]. It was observed that the use of a neurodevolpmental approach, focusing on normalized tone and movement pattern, is necessary to improve remaining motor function [9].

Rehabilitation represents an active problem solving and educational process, focused on disability and aiming to maximize the patient's participation in society, considering functional limitations and environmental and personal factors. The rehabilitative treatment may positively influence biological mechanisms underlying recovery of motor function, especially of upper limb.

Occupational therapy and physiotherapy, commonly used in the rehabilitative treatment, rarely lead to significant results in the grasping a hand-held object, finger and wrist extension and reaching gripper fingers [10]. For this reason, the stroke rehabilitation program is presented to illustrate classical and new approaches to stroke rehabilitation, and the potential of robot-assisted devices. Technologies are designed to improve sensory and motor function and repair brain function. In addition to post stroke cytolysis disuse, the distribution of type I and type II muscle fibers changes in affected muscles. Functional Electrical Stimulation (FES) is used to produce a functionally useful movement, decreasing activity limitation caused by stroke [11]. Moe and others introduced the term functional electrical stimulation (FES) to describe the use of neuromuscular electrical stimulation to activate paralyzed muscles in precise sequence and magnitude so as to directly accomplish functional tasks. Peckham et al. have demonstrated that chronic electrical stimulation facilitates reversal of fiber type conversion secondary to motor unit plasticity [12]. The therapeutic effect of FES is motor relearning, as the recovery of previously learned motor skills that have been lost following localized damage to the central nervous system. An important application of FES is represented by stroke [13]. Its effect can be exploited by stimulating the antagonist muscle to the spastic muscle [14]. Sub motoric stimulation of sensitive fibers has the effect of reducing the muscle tone and to aim sensor motor reorganization [15]. The location of central damage influences the therapeutic effect of electrical stimulation. In fact, Sonde et al. reported that, in patients whose basal ganglia were not affected by the lesion, electrical stimulation could have a good effect, whereas no therapeutic effect was achieved in cases of lesions of the periventricular white matter [16]. A study by Kawashima demonstrated a reduction in arm spasticity in patients after stroke, as indicated by the reduction of $\mathrm{H}$ reflex in the wrist flexor muscle and decreased Modified Ashworth Scale scores [17].

Additionally, Botulinum Toxin type A injection represents the gold standard therapy for focal spasticity after stroke in the upper and lower limbs [18]. BTX-A is a protein produced by the Clostridium Botulinum and seven botulinum toxins types have been recognized, from A to G. Botulinum toxin A cleave synaptosomal associated protein (SNAP-25), involved in vesicle fusion and mediating release of acetylcholine from axon ending. So, it causes flaccid paralysis of muscles. The therapeutic effects are transient with a mean duration of improvement approximately 3 months, caused by axonal sprouting. The most frequent adverse reactions to BTX-A are loss of clinical effect (especially antibodies will developed), loss of strength, muscle weakness and serious effects, including problems breathing or swallowing and spread to toxin effects [19]. The effect of combined use of BTX-A and FES in the treatment of spasticity was studied in animals. It was observed that the activity of botulinum toxin, with functional electric stimulation as an adjunctive therapy, was very important to reduce the neurogenic component of spasticity and to increase calf inhibition and provide a mechanical stretching effect while strengthening the stimulate muscles [20]. Many case studies has been chosen to illustrate the combined effects of lower limb [21] FES or upper limb [22] FES and BTX-A therapy in children with spastic cerebral palsy, but in the absence of a control group in both trials. Recently, Tsuchiya $\mathrm{M}$ et al. demonstrated for the first time the effect of combined use of BTX-A and FES in the treatment of spasticity in adults; they showed an improvement of active function in paretic hand [23].

Aim of this study is to assess if adding FES to conventional therapy such as botulinumtoxinA (BTX-A) and occupational therapy (OT) can improve spasticity in stroke patients.

\section{MATERIALS AND METHODS}

\section{Study Population}

All participants provided written informed consent before participating in the study.

36 patients (middle aged 55,25 $\pm 6,5$ ) were treated.

Inclusion criteria were:

- age between 40 and 70 years old;

- spasticity of the upper limbs for more than six months;

- spasticity of hand's muscle Flexor Digitorum Superficialis (FDS) with Modified Ashworth Scale $\geq 2$;

- no previous treatment with botulinumtoxinA or concomitant use of muscle relaxants

- no muscles fibrosis, tendon retractions or locking of the wrist joint, the metacarpophalangeal joint and the interphalangeal joint;

- no tumors at the site of electrical stimulation

- no dementia, different types of neuropathies, cardiac pacemaker or metallic prosthesis

- no anamnesis for epilepsy.

The patients recruited were randomly divided into two groups that were homogeneous. There were no statistically significant differences in age (middle age of 56,2 $\pm 5,5$ in I group and middle aged of $55,5 \pm 6,7)$, gender $(51 \%$ male and $49 \%$ female), site of injury (50\% dominant and $50 \%$ nondominant side in both groups) and severity of the spasticity.

The first group (Group I) consists of 18 patients and they were treated with incobotulinumtoxinA (Xeomin ${ }^{\circ}$, Merz), OT and FES; the second group (Group II) consists of 18 pa- 
tients and they were treated with incobotulinumtoxinA (Xeomin ${ }^{\circledR}$, Merz) and OT.

\section{Study Treatment}

We used vials of incobotulinum toxin A (Xeomin ${ }^{\circledR}$, Merz). One vial contains 100 units of Clostridium Botulinum neurotoxin type A. The dilution was $1 \mathrm{cc}$ into 1 standard vial of Xeomin®. We treated FDS muscle (ultrasound guide) with a middle dosage of $118,1 \pm 33,9$ in the first group and $116,4 \pm 35,6$ in the second one. The number of injection sites was two. Occupational therapy program consisted in exercises described in literature [24]. We used open fingers program of FES, with a frequency of $1 \mathrm{~Hz}$ and an intensity of 6/9.

\section{Assessment and Outcome Measures}

All patients were evaluated with the Modified Ashworth Scale, with the measurement of the ROM, the evaluation of the amplitude of the compound action potential of the median nerve (registration site in the flexor digitorum superficial) and ARAT test at the time T0 (recruitment and infiltration), T1 (20 days after first infiltration), T2 (3 months after first infiltration), T3 (four months after first infiltration; we reinjected botulinumtoxinA), T4 (20 days after second infiltration), T5 (3 months after second infiltration), T6 ( four months after first infiltration; we reinjected botulinum toxin A).

The assessors of the outcome parameter were blinded with respect to the allocated intervention.

Modified Ashworth Scale (MAS) of FDS was evaluated with difficulty to stretch fingers. Passive range of motion (ROM) of metacarpophalangeal and interphalangeal joints was assessed using a goniometry. We used Action Research Arm Test (ARAT) to measure hand's performance. We also evaluated the median nerve electroneurography (ENG) to determine the amplitude of the compound muscular action potential (cMAP) of the FDS. The neurographic examination was performed using Nicolet Viking 8-channel. Before the procedure, patients were placed in the supine position, with the forearm fully supinated, the elbow extended and the hand face up. Recording electrode (bipolar concentric needle, diameter $26 \mathrm{G}$, recording area $0,07 \mathrm{~mm} 2$ ) was placed over the muscle belly (FDS), a ground electrode was placed between the stimulating and recording electrodes. The first stimulation was performed at the elbow and the frequency is $1 \mathrm{~Hz}$. To achieve supramaximal stimulation, current intensity was slowly increased until it reached a level at which the recorded potential does not increase. cMAP amplitude was measured from negative peak to positive peak (peak to peak) and expressed in mill volts, using antidromic technique. The low frequency cutoff (high pass filter) was set at $2-5 \mathrm{~Hz}$ and the high frequency cutoff (low pass filter) was set at 10-20 Hz.

All patients have carried out an occupational therapy daily for 30 days after the injection of botulinum toxin, then three times a week.

In the first group, patients were treated with an individualized program using the NESS $\mathrm{H} 200^{\mathrm{TM}}$ device (originally Handmaste). The NESS H200 device is a portable, non invasive wrist hand orthotics utilizing microprocessor controlled muscle activation. It features five surface electrodes, used to deliver electrical stimulation, via the skin, to the motor nerves. It is possible a varying sequence of movements designed to imitate the natural motion of the hand, according to the patient's need and goals. The frequencies of electrical stimulation used can vary widely, in order to avoid fatigue, discomfort or pain (when sensitivity is retained) [25].

Electrical stimulation devices deliver pulses in waveform patterns that are often represented by geometric shapes. These shapes characterize electrical current that rises above a zero baseline for the extent of the stimulation paradigm (uniphasic: direct current) or current that alternates above and below the baseline (biphasic or alternating current). Balanced biphasic waveforms are most reported to be most comfortable, generate greater torque with muscle contraction and pose the least risk for skin reaction. It is well known that endurance exercise readily promotes fiber type transformation, from a glycolytic to an oxidative phenotype; conversely, decreases of the habitual activity level due to injury result in decreased oxidative capacity, force output and fatigue resistance [26].

The total length of the study was about 8 months.

\section{Statistical Analysis}

The age and the value of cMAP can be considered distributed according to Gauss, therefore the data are summarized as mean and standard deviation; the analysis of the effectiveness of treatment and the comparison between the different points of follow-up was made by applying a model of analysis of variance for repeated measures. In order to evaluate the effect of some covariates such as sex, age (divided into classes $>65,<=64$ ), the location of the lesion and were included as random effects in the model, but excluded from the final model because it found no statistically significant $(\mathrm{p}<0,05)$.

The other quantitative variables (Ashworth scale, clinical outcome measure, results goniometric) instead are acceptably distributed according to Gauss, therefore the data were summarized as median and range; comparing therapies and between the different moments of follow-up were performed with nonparametric methods (Kruskal-Wallis and Friedman test based on what is most appropriate). The post hoc comparisons were performed by applying the Bonferroni correction.

The qualitative variables were summarized as counts and percentages and comparison between independent samples were performed using the chi-square test. The assessment of the correctness of the recruitment and then the comparability of the two groups was performed using the student t-test and the Wilcoxon test as appropriate for quantitative variables and the chi-square test for qualitative variables. Differences between groups were considered statistically significant when the $\mathrm{p}$-value was less than 0.05 . The comparison for subgroups was conducted by applying the Bonferroni correction; in relation to the number of comparisons of interest for the search target was considered as statistically significant a value of $p$-value $<0.0045$. The analysis was conducted using the SAS 9.3 software for PC; Friedman's test was conducted using the statistical software R version 12 .

\section{RESULTS}

The first group (Group I) treated with BTX-A, FES and OT showed a statistically significant improvement than the other group (Group II) treated with BTX-A and OT. We demonstrated 
a reduction of spastic upper limb assessed by Modified Ashworth Scale (Fig. 1, p <0.001), an increase of range of motion (ROM) of metacarpophalangeal joints and interphalangeal joints (Fig. 2, p <0.001), a reduction of amplitude of the compound muscular action potential (cMAP) of the FDS (Fig. 3, p <0.001) and an increase of performance assessment using the Action Research Arm Test (ARAT; Fig. 4, p<0.001) in group I for 4 months after each infiltration.

\section{DISCUSSION}

The exercise based on muscle recruitment especially volunteer is considered a key element in promoting recovery after injury ictal acute central nervous system, increasing the production of trophic factor in selected brain regions and, especially, modulating crucial aspects of neuroplasticity [2728]. The use of a robot-assisted rehabilitation therapy allows the quantification of input sensory-motor and the increase of outcome motor [29]. Recent studies on robotic therapy encourage their use, especially as patients undergoing this technique show a significant increase in voluntary activity than results obtained without the use of robotic equipment. This supports the hypothesis that the robotic therapy facilitates driving, increasing and stimulating the process probably neuro-plastic on motor learning. The robotic devices interact with the patient in real time, to allow the mobilization of a limb as much as any inert assisted neuro therapy facilitate[30]. The main motivation to the use of the FES is the fact that many research groups in the field of neurorehabilitation have concluded that the greater the training and exercise motor repeated by the patient affected by stroke, the better the chance of recovery and reacquisition of motor sequences that can be lost as a result of the disease. The longer the patient deals with a motor structured training, the better the chances of recovery [31-32]. Using a robot-assisted neurorehabilitation protocol will therefore: increase the actual time devoted to the exercise; promote brain plasticity, so that other areas of the brain take part in the execution of the movements; and make more effective the action of the physiotherapist [33-34].

This study, according to Tsuchiya M, demonstrated[19] that combined treatment TB - OT - FES improved muscle tone and then the entire upper limb spasticity with improvement of joint ROM at the metacarpophalangeal and interphalangeal joints with a greater recovery of grades and a greater extension of the fingers which resulted in a better functional recovery of the function of taking of the paretic hand, thus allowing a minimum of movements such as to enable an improvement in personal hygiene and a minimum of autonomy in some activities of daily living which has also been observed all'ARAT test. Examination electroneurography cMAP also showed persistence of action of botulinum toxin in the first group; so, combined treatment allow the time to lengthen the interval between sessions of inoculation of botulinum toxin thereby reducing the possibility of occur-

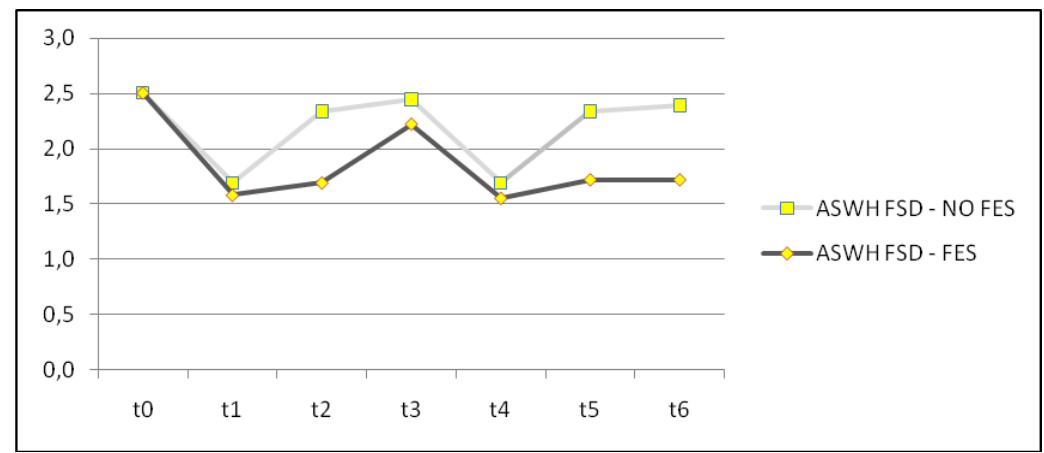

Fig. (1). MAS modification in the two groups (with and without FES) at different times.

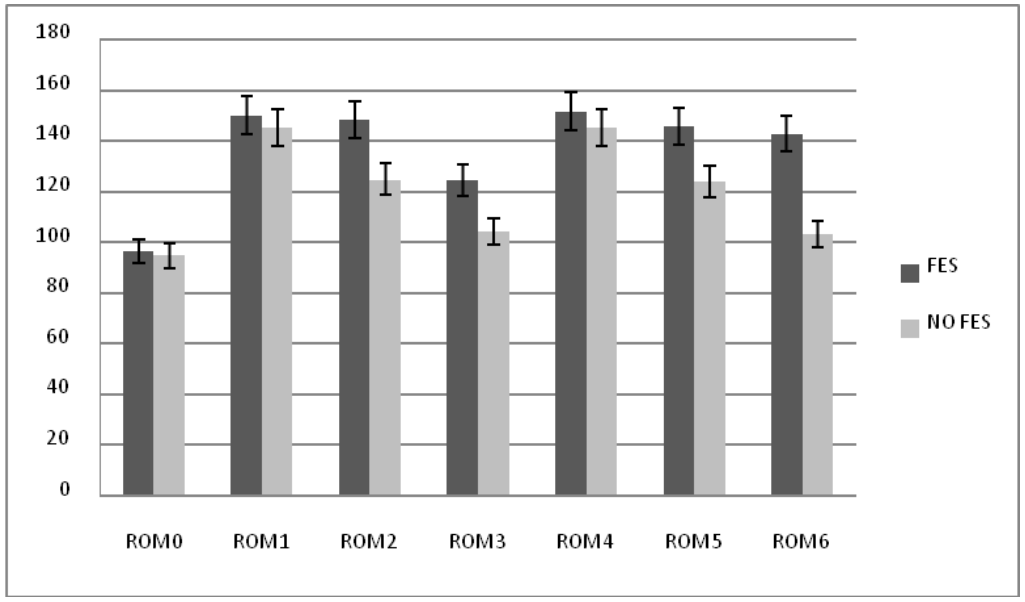

Fig. (2). Variations of passive ROM of the fingers in the two groups at different times of analysis 


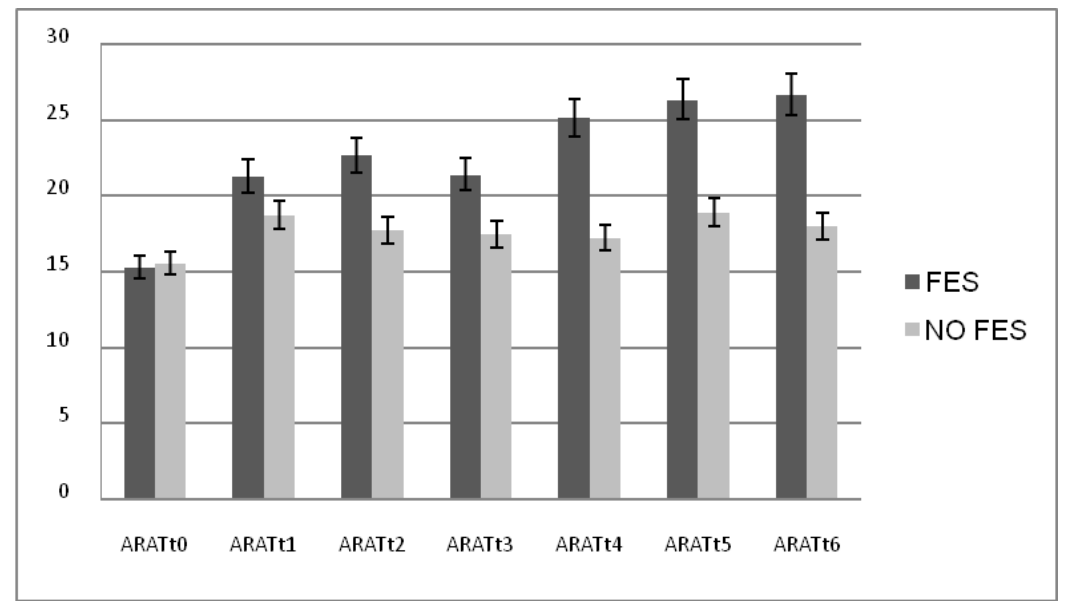

Fig. (3). ARAT test scores in the two groups at different timepoints.

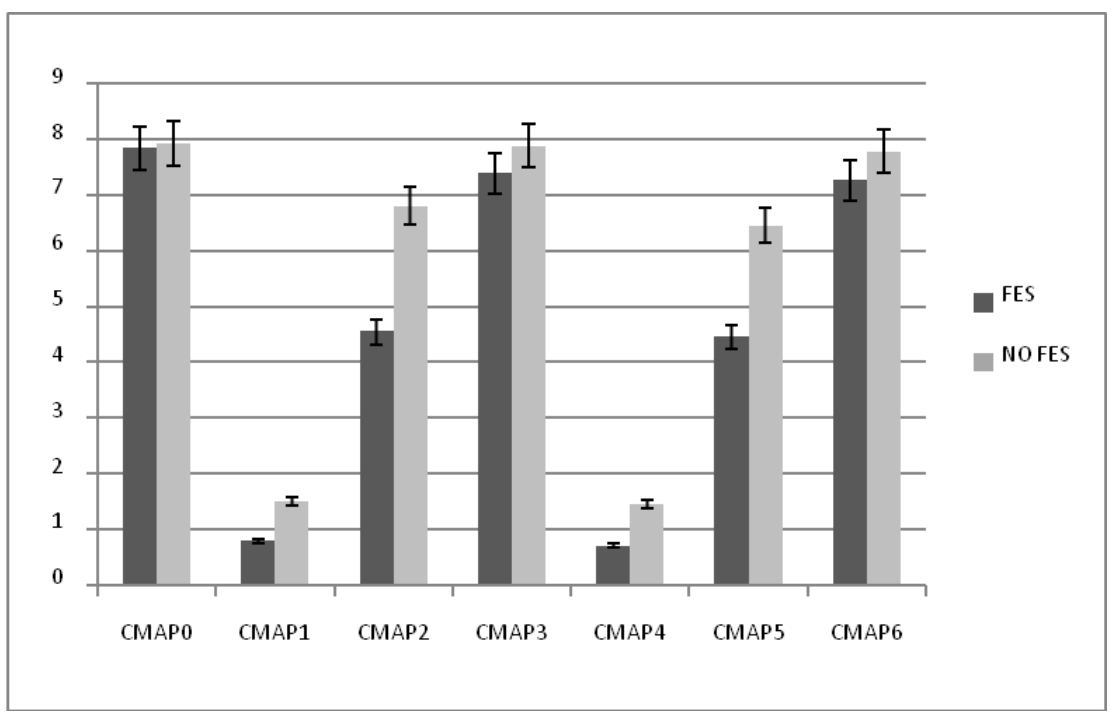

Fig. (4). Median nerve cMAP recorded in muscle FDS at different times in the two groups

rence of resistance mediated[19]. The study, therefore demonstrates that the combination of infiltration with Botulinum Toxin, rehabilitation treatment on a daily basis and then three times a week and FES is essential for effective results and functional recovery of the upper limb spastic. The combination of the three treatments interrupts the sequence of pathological events that contribute to the modification of the passive mechanical properties of the muscle, with a net increase of the resistances elastic and plastic and with rearrangement in the sense tonic muscle in phase of spasticity. Furthermore, the possibility to release easily the limb suffering from spasticity has a specific effect on the connective tissue and the capillary network; the latter in fact tends to increase, while the amount of connective tissue tends to stabilize. The application of the FES flanked to treatment with botulinum toxin and OT therefore it demonstrates an effective synergy for a greater functional recovery of the upper limb, which is detected in increasing agility by the patient in performing certain fine movements with the fingers as for example the gripper or the grasp; this leads to greater independence in activities of daily living and a greater participa- tion of individuals in social life and relationships with significant benefits in the mood.

\section{CONCLUSION}

The simultaneous therapy of FES-botulinum toxin type A-OT proves effective in a faster functional recovery of the upper limb in patients post stroke, obtaining an increasing of fine movements of hand of these patients.

\section{CONFLICT OF INTEREST}

The authors confirm that this article content has no conflict of interest.

\section{ACKNOWLEDGEMENTS}

Declared none.

\section{REFERENCES}

[1] Picelli A, Tamburin S, Dambruoso F, Midiri A, Girardi P, Santamato A, Fiore P,Smania N. Topical distribution of initial paresis of the limbs to predict clinically relevant spasticity after ischemic 
stroke: a retrospective cohort study. Eur J Phys Rehabil Med. 2013 Dec 17. [Epub ahead of print] PubMed PMID: 24346154.

[2] Sommerfeld DK, Eek EU, Svensson AK, Holmqvist LW, von Arbin MH. Spasticity after stroke: its occurrence and association with motor impairments and activity limitations. Stroke. 2004 Jan;35(1):134-9. Epub 2003 Dec 18. PubMed PMID: 14684785.

[3] Lundström E, Smits A, Terént A, Borg J. Time-course and determinants of spasticity during the first six months following first-ever stroke. J Rehabil Med. 2010 Apr;42(4):296-301. doi: 10.2340/16501977-0509. PubMed PMID: 20461330

[4] Lance JW. The control of muscle tone, reflexes, and movement: Robert Wartenberg lecture. Neurology. 1980; 30: 1303-1313.

[5] Watkins CL, Leathley MJ, Gregson JM, Moore AP, Smith TL, Sharma AK. Prevalence of spasticity post stroke. Clin Rehabil. 2002 Aug;16(5):515-22. PubMed PMID:12194622.

[6] Hurst W. The Heart, Arteries and Veins. 10. New York, NY: McGraw-Hill; 2002

[7] Wolf SL, Lecraw DE, Barton LA, Jann BB. Forced use of hemiplegic upper extremities to reverse the effect of learned nonuse among chronic stroke and head-injured patients. Exp Neurol. 1989:104(2): 125-32.

[8] Wade DT, Collin C. The Barthel ADL Index: a standard measure of physical disability? Int Disabil Stud. 1988; 10: 64-67.

[9] Lennon S. The Bobath concept: a critical review of the theoretical assumptions that guide physiotherapy practice in stroke rehabilitation. Phys Ther Rev. 1996; 1: 35-45.

[10] Hara Y. Neurorehabilitation with new functional electrical stimulation for hemiparetic upper extremity in stroke patients. J Nippon Med Sch. 2008 Feb;75(1):4-14. Review. PubMed PMID: 18360073

[11] Chen F, Qi Z, Luo Y, Hinchliffe T, Ding G, Xia Y, Ji X. Nonpharmaceutical therapies for stroke: Mechanisms and clinical implications. Prog Neurobiol. 2014 Jan 7. doi: 10.1016 / j.pneurobio.2013.12.007. [Epub ahead of print] PubMed PMID: 24407111

[12] Peckham PH, Mortimer JT, Marsolais E B. Alteration in the force and fatigability of skeletal muscle in quadriplegic humans following exercise induced by chronic electrical stimulation. Clin Orthop 1976; 114: 326-334

[13] Schuhfried O, Crevenna R, Fialka-Moser V, Paternostro-Sluga T. Non-invasive neuromuscular electrical stimulation in patients with central nervous system lesions: an educational review. J Rehabil Med. 2012 Feb;44(2):99-105. doi: 10.2340/16501977-0941. Review. PubMed PMID: 22334346

[14] Peurala SH, Pitkänen K, Sivenius J, Tarkka IM. Cutaneous electrical stimulation may enhance sensorimotor recovery in chronic stroke. Clin Rehabil 2000; 16: 709-716.

[15] Sonde L, Bronge L, Kalimo H, Viitanen M. Can the site of brain lesion predict improved motor function after low-TENS treatment on the post-stroke paretic arm? Clin Rehabil 2001; 15: 545-551.

[16] Kawashima N, Popovic MR, Zivanovic V. Effect of intensive functional electrical stimulation therapy on upper-limb motor recovery after stroke: case study of a patient with chronic stroke. Physiother Can. 2013 Winter;65(1):20-8. doi: 10.3138/ptc.2011-36. PubMed PMID: 24381377; PubMed Central PMCID: PMC3563372

[17] Wilkenfeld AJ. Review of electrical stimulation, botulinum toxin, and their combination for spastic drop foot. J Rehabil Res Dev. 2013 Jun;50(3):315-26. PubMed PMID: 23881758.

[18] Kaku M, Simpson DM. Spotlight on botulinum toxin and its potential in the treatment of stroke-related spasticity. Drug Des Devel Ther. 2016 Mar 8;10:1085-99.

[19] Tsuchiya M, Morita A, Hara Y. Effect of Dual Therapy with Botulinum Toxin A Injection and Electromyography-controlled Func- tional Electrical Stimulation on Active Function in the Spastic Paretic Hand. J Nippon Med Sch. 2016;83(1):15-23.

[20] Sheffler LR, Chae J. Neuromuscular electrical stimulation in neurorehabilitation. Muscle Nerve. 2007 May;35(5):562-90. Review. PubMed PMID: 17299744

[21] Galen S, Wiggins L, McWilliam R, Granat M. A combination of Botulinum Toxin A therapy and Functional Electrical Stimulation in children with cerebral palsy--a pilot study. Technol Health Care. 2012;20(1):1-9. doi: 10.3233/THC-2011-0648. PubMed PMID: 22297709

[22] Pieber K, Herceg M, Wick F, Grim-Stieger M, Bernert G, Paternostro-Sluga T. Functional electrical stimulation combined with botulinum toxin type A to improve hand function in children with spastic hemiparesis - a pilot study. Wien Klin Wochenschr. 2011 Feb;123(3-4):100-5. doi: 10.1007/s00508-010-1518-7. Epub 2011 Jan 19. PubMed PMID: 21240688.

[23] Chang MA. Possible Adverse Effects of Repeated Botulinum Toxin A Injections to Decrease Post-Stroke Spasticity in Adults Undergoing Rehabilitation: A Review of the Literature. J Allied Health. 2015;44(3):140-4.

[24] Demetrios M, Brand C, Louie J, Khan F. More than a black box of rehabilitation: Characterizing therapy programmes following botulinum toxin injections for spasticity in adults with stroke. J Rehabil Med. 2016 Apr 28;48(5):426-34.

[25] Gorman PH. An update on functional electrical stimulation after spinal cord injury. Neurorehabil Neural Repair. 2000;14(4):251-63.

[26] Ragnarsson KT. Functional electrical stimulation after spinal cord injury: current use, therapeutic effects and future directions. Spinal Cord. 2008 Apr;46(4):255-74. Epub 2007 Sep 11.

[27] Gómez-Pinilla F., Ying Z., Roy RR., Molteni R., Edgerton VR. Voluntary exercise induces a BDNF-mediated mechanism that promotes neuroplasticity. $J$ Neurophysiol. 2002 Nov;88(5):218795.

[28] Fritsch N., Gentile A., Kearns K., Benois A., Fontaine B. Investigation about a lactic acidosis. Ann Fr Anesth Reanim. 2010 Dec; 29(12):925-6. doi: 10.1016/j.annfar.2010.10.006.

[29] Volpe BT., Krebs HI., Hogan N., Edelstein L., Diels C., Aisen M. A novel approach to stroke rehabilitation: robot-aided sensorimotor stimulation. Neurology. 2000 May 23;54(10):1938-44.

[30] Weber DJ, Skidmore ER, Niyonkuru C, Chang CL, Huber LM, Munin MC. Cyclic functional electrical stimulation does not enhance gains in hand grasp function when used as an adjunct to onabotulinumtoxinA and task practice therapy: a single-blind, randomized controlled pilot study. Arch Phys Med Rehabil. 2010, May;91(5):679-86. doi: 10.1016/j.apmr.2010.01.010.

[31] Kapadia NM, Nagai MK, Zivanovic V, Bernstein J, Woodhouse J, Rumney P, Popovic MR. Functional Electrical Stimulation Therapy for Recovery of Reaching and Grasping in Severe Chronic Pediatric Stroke Patients. J Child Neurol. 2013 Apr 12. [Epub ahead of print] PubMed PMID: 23584687.

[32] H.Weingarden, H. Ring. Functional electrical stimulation-induced neural changes and recovery after stroke. Eura medicophys $\mathbf{2 0 0 6}$

[33] Caleo M. Rehabilitation and plasticity following stroke: Insights from rodent models. Neuroscience. 2015 Dec 17;311:180-94. doi: 10.1016/j.neuroscience.2015.10.029. Epub 2015 Oct 19.

[34] Oza CS., Giszter SF. Trunk robot rehabilitation training with active stepping reorganizes and enriches trunk motor cortex representations in spinal transected rats. $J$ Neurosci. 2015 May 6; 35(18): 7174-89. doi: 10.1523 /JNEUROSCI.4366-14.2015. 